\title{
OTIONOMTCS
}

Revista de economía, empresa y sociedad

\section{H porqué de un dosier sobre prevención de riesgos laborales}

\section{Kavier Baraza Sánchez y Mar Sabadell i Bosh}

Profesores de los Estudios de Economía y Empresa (UOC) y editores del dosier «Prevención de riesgos laborales: tendencias en tiempos de crisis»

Según la Estadística de accidentes de trabajo del Ministerio de Empleo y Seguridad Social para el año 2014, en Cataluña se produjeron 91.978 accidentes, de los que 14.969 fueron in itinere. Del total, 699 fueron graves y 83 mortales. Esta situación, si bien presenta mejoría respecto a años anteriores, sigue comportando un factor relevante en el día a día de la actividad empresarial, tanto a nivel emocional y de seguridad de sus trabajadores como de costes directos e indirectos sobre el presupuesto de la empresa.

Estos datos, junto con la celebración del XX aniversario de la Ley de Prevención de Riesgos Laborales (PRL), hacen que sea un buen momento para dedicar un dosier a la PRL desde la óptica de su estrecha relación con la empresa, más aún cuando la situación de crisis acumulada en los últimos años ha tenido una clara influencia sobre las tasas de accidentabilidad y el cuidado de la seguridad y salud de los trabajadores. Esta ley marcó un cambio sustancial en la conceptualización de la prevención de riesgos apostando por la integración de la acción preventiva en la gestión de los organizaciones con el fin de minimizar la accidentalidad laboral y las enfermedades profesionales. Ahora que se cumplen los 20 años es el momento de dar un nuevo paso importante, evolucionando del concepto de PRL hacia el de organizaciones saludables.

El presente dosier es el fruto de la colaboración de diversos expertos que han aportado su experiencia y conocimiento en este número de la revista Oikonomics, de los Estudios de Economía y Empresa de la UOC.

En el primer artículo, Jaume de Montserrat, del Instituto de Seguridad y Salud de la Generalitat de Cataluña, junto a otros profesionales del organismo, aportan una información relevante sobre la accidentabilidad que ha sufrido Cataluña desde el inicio de la crisis en el año 2008 y analizan su impacto en el deterioro de las condiciones de seguridad y salud en el trabajo.

El segundo artículo, escrito por Carles Salas, profesor del Departamento de Organización de Empresas de la Universidad Politécnica de Cataluña y responsable de Seguridad y Salud Laboral de TUSGSAL, aborda la necesidad de utilizar indicadores socioeconómicos en el ámbito preventivo como herramienta para la buena gestión de la PRL, incluyendo para ello la argumentación rentabilista. En este sentido, Carles Salas nos introduce en el cálculo de los costes de la accidentabilidad como punto de partida para un buen análisis coste-beneficio.

Manuel Bestratén, consejero técnico de dirección del Instituto Nacional de Seguridad e Higiene en el Trabajo (INSHT) y experto en Gestión de la PRL, nos presenta en el tercer artículo del dosier la relación existente entre la PRL y la responsabilidad social empresarial, y muestra, a partir de un estudio realizado por el INSHT, cómo la PRL actúa de motor de cambio en aquellas empresas socialmente responsables.

En el cuarto artículo, Eva Rimbau, profesora de los Estudios de Economía y Empresa de la UOC, describe los diferentes tipos de liderazgo según su impacto sobre el estrés laboral, patología cada día más presente en el entorno laboral. Para ello, aborda la incidencia que el modelo de liderazgo puede tener sobre el bienestar psi- 
cológico de los trabajadores y las posibles herramientas y comportamientos que los mandos han de tener para minimizar el impacto negativo.

Mar Sabadell, abogado y profesora de los Estudios de Economía y Empresa de la UOC, y Guillermo García, abogado y profesor de la Universidad Internacional de la Rioja, se aproximan a la prevención desde el punto de vista legal. Tras veinte años de vigencia de la ley de PRL, se ha consolidado el enfoque preventivo de la seguridad y salud laboral, pero se abre una nueva etapa que supone importantes retos jurídicos y la necesidad de regenerar las prácticas preventivas frente a una nueva manera de trabajar en la que ciertos elementos clave del contrato de trabajo no se presentan en la forma tradicional. El artículo aborda como cohonestar las obligaciones preventivas en el teletrabajo y no perjudicar el derecho a la seguridad y salud laboral.

Ines Dalmau, colaboradora de la UOC en el ámbito de la Psicosociología, y Ramon Ferrer, profesor del Departamento de Metodología de las Ciencias del Comportamiento de la Universidad de Barcelona, analizan en el sexto artículo del dosier el modelo de teletrabajo o trabajo virtual, cada vez más presente en la sociedad actual, no solo desde el punto de vista de sus ventajas sino haciendo un exhaustivo análisis de las situaciones de riesgo laboral que comporta, en especial en cuanto a riesgos psicosociales.

Finalmente, Agustín Sánchez-Toledo, colaborador del máster de Responsabilidad social corporativa de la UOC, y Xavier Baraza, director del máster de Prevención de riesgos laborales, analizan en el último artículo del dosier los contenidos y la necesidad de implantación de la Norma ISO 39001, sobre gestión de la seguridad vial, en las diferentes organizaciones a fin de hacer frente a la elevada accidentabilidad laboral en los desplazamientos al puesto de trabajo.

Complementa esta edición de la revista Oikonomics un artículo del profesor de los Estudios de Economía y Empresa de la UOC Francesc González sobre la realidad aumentada y su aplicación específica a un ámbito tan relevante como el sector turístico, tema de vigente actualidad. La presente edición de la revista incluye también la publicación de uno de los mejores trabajos finales de grado de los estudiantes de los Estudios de Economía y Empresa de la UOC. Se presenta en este caso el plan de empresa del alumno del grado de ADE Daniel Macías Perea, bajo el título «Classyfied, la compra más fácil».

Esperamos que el esfuerzo puesto en esta nueva entrega de Oikonomics, la revista de los Estudios de Economía y Empresa de la UOC, os permita adentraros en el mundo de la prevención de riesgos laborales desde la óptica del mundo empresarial.

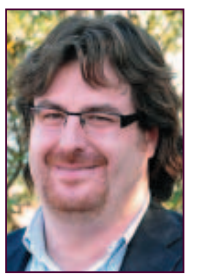

\section{Xavier Baraza Sánchez jbaraza@uoc.edu Profesor de los Estudios de Economía y Empresa (UOC)}

Ingeniero Químico Industrial y doctor en Ingeniería del Medio Ambiente y del Producto por la Universidad de Barcelona. Máster en Prevención de riesgos laborales (tres especialidades) por la Universidad Politécnica de Cataluña y máster en Dirección y administración de empresas por la Escuela Europea de Negocios. Ha sido director de Seguridad, salud y medio ambiente de una empresa del sector de la química fina entre los años 2005 y 2010. Actualmente es profesor de los Estudios de Economía y Empresa de la Universitat Oberta de Catalunya y director del máster universitario de Prevención de riesgos laborales de esta universidad. Autor de varios libros en materia de PRL y de artículos en revistas de referencia. Miembro del grupo de investigación consolidado DigiBiz (Digital Business Research Group). 


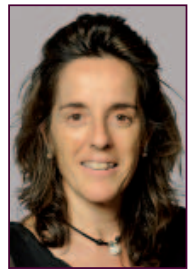

Mar Sabadell i Bosch

msabadell@uoc.edu

Profesora de los Estudios de Economía y Empresa (UOC)

Abogado, licenciada en Derecho por la Universidad de Barcelona y máster en Sociedad de la información y el conocimiento por la Universitat Oberta de Catalunya. Desde 1999 es profesora de los Estudios de Economía y Empresa de la UOC, donde imparte actualmente docencia, y durante los años 2006-2009 ha desarrollado el cargo de adjunta al vicerrector de Ordenación Académica y Profesorado.

Es miembro del grupo de investigación consolidado DigiBiz (Digital Business Research Group), adscrito al IN3. Sus intereses en investigación se centran actualmente en el estudio de la interrelación entre el uso de las TIC y el trabajo, y en el desarrollo de formas de trabajo flexible y trabajo a distancia, con una especial inquietud en materia de responsabilidad empresarial en la prevención de riesgos asociados al teletrabajo.

Los textos publicados en esta revista están -si no se indica lo contrario- bajo una licencia Reconocimiento-Sin obras derivadas 3.0 España de Creative Commons. Puede copiarlos, distribuirlos y comunicarlos públicamente siempre que cite su autor y la revista y la institución que los publica (autoría, nombre de la revista, institución editora); no haga con ellos obras derivadas. La licencia completa se puede consultar en http://creativecommons.org/licenses/by-nd/3.0/es/deed.es.

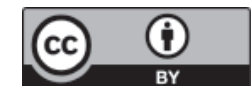

